\title{
CDISC SDTM Relation to Reference Period Terminology
}

National Cancer Institute

\section{Source}

National Cancer Institute. CDISC SDTM Relation to Reference Period Terminology. NCI

Thesaurus. Code C66728.

Terminology codelist used with Relation to Reference Period within the Clinical Data

Interchange Standards Consortium Study Data Tabulation Model. 